INPLASY

PROTOCOL

To cite: Moreno et al. Handeye laterality profiles. Does affect sports performance? A systematical review. Inplasy protocol 2020110127. doi: 10.37766/inplasy2020.11.0127

Received: 28 November 2020

Published: 28 November 2020

Corresponding author: Miquel Moreno

miquel.moreno2@e-campus.uab.cat

Author Affiliation:

Department of Basic, Evolutive and Educational Psychology.

Universitat Autònoma de

Barcelona

Support: Grants from MINECO \& FEDER.

Review Stage at time of this submission: Preliminary searches.

Conflicts of interest: None.

\section{Hand-eye laterality profiles. Does affect sports performance? A systematical review}

Moreno, M¹; Losilla, JM²; Capdevila, L³.

Review question / Objective: Prevalence of hand-eye laterality profiles in different sports modalities and its relationship with sports performance and psychological factors.

Condition being studied: Hand-eye laterality profiles in the sport practice, both in general population and in athletes. Hand-eye laterality may be related to performance in some sports modalities, as well as to psychological factors.

Information sources: Literature searching will be done using the following databases in the indicated order and form the indicated portals: PsycINFO by EBSCOhost, Scopus by Elsevier, Medline by Pubmed, Dissertations \& Theses Global by ProQuest. To include grey literature, we also searched in Google and reviewed up to 100 links. In addition, search alerts will be established for PsycINFO and Scopus until December 2020. The search strategy will follow the Peer Review of Electronic Search Strategies (PRESS) guidelines recommendations (McGowan et al., 2016).

INPLASY registration number: This protocol was registered with the International Platform of Registered Systematic Review and Meta-Analysis Protocols (INPLASY) on 28 November 2020 and was last updated on 28 November 2020 (registration number INPLASY2020110127).

\section{INTRODUCTION}

Review question / Objective: Prevalence of hand-eye laterality profiles in different sports modalities and its relationship with sports performance and psychological factors.
Rationale: Hand-eye laterality profile consists in having the same side of preference for both hand and eye (uncrossed profile, UCP), or having eye and hand preference at different sides of the body (crossed profile, CP). Although it has been studied for centuries (Porta, 1593), laterality is a concept which still leaves 
some relevant questions to solve. Previous research has already indicated the relevance of the laterality study for the detection and guidance of talented young athletes (Laborde et al., 2009), the improvement in sports performance (Dorochenko, 2013; Porac and Coren, 1981), or more specifically the prevalence of UCP and CP laterality in professional tennis players (Ziagkas et al., 2018). Some trends of experts in tennis training also point to a relationship between hand-eye laterality and the tactical behavior of the best players in the world (Dorochenko, 2013). However, some authors point out that the available studies on hand-eye laterality report contradictory results, both on the prevalence of cross-laterality, and on the impact of hand-eye laterality on sports performance in the different sports modalities or its association with psychological factors (e.g., Robinson et al., 1997).

Condition being studied: Hand-eye laterality profiles in the sport practice, both in general population and in athletes. Hand-eye laterality may be related to performance in some sports modalities, as well as to psychological factors.

\section{METHODS}

Search strategy: The search strategy will consist on a long string conformed by the different terms and synonyms related with hand-eye laterality combined with the sport field: ("eye-hand dominance" OR "eyehand laterality" OR "hand-eye dominance" OR "hand-eye laterality" OR "crossed laterality"[ OR "cross laterality"OR "contralateral dominance" OR "crosslateral dominance" OR "crosslateral dominance" OR "homogeneous laterality" OR "homogeneous dominance" OR "homogeneous preference" OR "ipsilateral preference" OR "ipsilateral dominance" OR "cross dominance" OR "crossed dominance" OR "hand-eye coordination" OR "eye-hand-coordination" OR "hand-eye preference" OR "eye-hand preference" OR "lateral preference" OR "lateral dominance" OR "cross-lateral dominance" OR "eyedness" OR "ocular dominance" OR "dominant eye" OR "ocular laterality" OR "handedness" OR "uncrossed dominance" OR "eye-hand coordination" OR "hand-eye preference" OR "eye-hand preference" OR "cross laterality" OR "uncrossed laterality" OR "left-handed" OR "left-handedness" OR "right-handed" OR "oculo-motor coordination") AND "sport*" This search strategy will be adapted to the syntax and specific characteristics of each bibliographic database: PsycINFO by EBSCOhost, Scopus by Elsevier, Medline by Pubmed, Dissertations \& Theses Global by ProQuest. The systematic search will be performed in November and December 2020, limited by language (English, French and Spanish) and with no time limitation.

Participant or population: Subjects at any sports context. No exclusion criteria will be applied by gender and age.

Intervention: The exposure of interest in this study is the hand-eye laterality in any sport modality.

\section{Comparator: None.}

Study designs to be included: Original empirical studies, with experimental (randomized or non-randomized), nonexperimental (observational longitudinal or cross-sectional) or single-case research designs.

Eligibility criteria: Eligible studies have to fulfill the following inclusion criteria: a) original empirical studies (experimental, quasi-experimental, observational or single-case designs), which b) collect direct information on hand-eye laterality. No exclusion criteria will be applied by gender, age or temporal limit of the publication.

Information sources: Literature searching will be done using the following databases in the indicated order and form the indicated portals: PsycINFO by EBSCOhost, Scopus by Elsevier, Medline by Pubmed, Dissertations \& Theses Global by ProQuest. To include grey literature, we also searched in Google and reviewed up to $\mathbf{1 0 0}$ links. In addition, search alerts will 
be established for PsycINFO and Scopus until December 2020. The search strategy will follow the Peer Review of Electronic Search Strategies (PRESS) guidelines recommendations (McGowan et al., 2016).

Main outcome(s): Prevalence of each type of hand-eye laterality concerning at the sport discipline. Relationship between hand-eye laterality and sports performance. Relationship between handeye laterality and psychological factors.

\section{Additional outcome(s): None.}

Data management: References identified by the search strategy will be entered into Mendeley bibliographic software, and duplicates will be removed. Titles and abstracts will be screened independently by two reviewers. When decisions are unable to be made from title and abstract alone, the full paper will be retrieved. Fulltext inclusion criteria will be screened independently by two reviewers. Discrepancies during the process will be resolved through discussion (with a third reviewer where necessary). A template will be designed to extract the data from the included articles, in order to assess the quality of the studies and synthesize their results. Extracted information will include: general information (author, title, year, journal, research design); sample information (size, age, sex, population/ country, sport discipline, etc.); laterality data (handedness test, eyedness test, cross-uncross distribution) and discussion information (differences between sports, relations laterality-performance, psychological treats, gender differences, skills analyzed, outstanding conclusions, related concepts). Data extraction will also be carried out independently by two reviewers.

Quality assessment / Risk of bias analysis: To maximize the reporting quality of the review we will follow the PRISMA Extension for Scoping Reviews (PRISMA-ScR) recommendations (Tricco et al., 2018). The following tools will be used to assess the risk of bias of the included studies: - To assess the risk of bias of experimental and quasi-experimental studies we will apply the Cochrane Collaborations' tool (Higgins et al., 2011). - To assess the risk of bias of observational studies (cohorts, casecontrol and cross-sectional) we will apply the ROBS tool (Losilla et al., 2018). - To assess the risk of bias of single-case studies we will apply the checklist proposed by Maggin et al. (2013); this tool is based on the evidence standards developed by the What Works Clearinghouse (WWC, Kratochwill et al., 2010) in the evaluation of single-subject research. Two independent reviewers will evaluate the risk of bias of each included study. Discrepancies in this evaluation process will be resolved through discussion (with a third reviewer where necessary).

Strategy of data synthesis: We will provide a narrative synthesis of the characteristics and results of the included studies. We will meta-analyze the prevalences (using observed proportions) of hand-eye laterality profiles and their relationship with sport performance and psychological factors (using standardized effect size indices, such as Cohen's de, Hedges' $g$ and Pearson's r). The most relevant data extracted will also be shown in tables and graphics.

Subgroup analysis: Hand-eye profiles prevalences and their relationship with sport performance and psychological factors will be classified according to type of hand-eye laterality, level of sport practice, sport discipline, handedness and eyedness test, and gender and age of study participants.

Sensibility analysis: Hand-eye profiles prevalence and their relationship with sport performance and psychological factors will be classified according to the year of data collection and research design and risk of bias of the primary studies.

Language: English.

Country(ies) involved: Spain.

Other relevant information: None. 
Keywords: Systematic review; hand-eye laterality; sports performance; crossed hand-eye laterality; uncrossed hand-eye laterality.

Dissemination plans: The results will be disseminated by its publication in a peerreviewed journal and presented at relevant conferences.

Contributions of each author:

Author 1 - Miquel Moreno - This author will actively participate in all phases of development of the review and the writing of the manuscript.

Email: miquel.moreno2@e-campus.uab.cat Author 2 - Josep Maria Losilla - This author will actively participate in all phases of development of the review and the writing of the manuscript.

Email: josepmaria.Losilla@uab.cat

Author 3 - Lluis Capdevila - This author will actively participate in all phases of development of the review and the writing of the manuscript.

Email: lluis.capdevila@uab.cat 